\title{
Exercise training improves memory and produces changes in the adrenal gland morphology in the experimental autoimmune encephalomyelitis
}

\author{
Muthanna HAFEDH ${ }^{1}$ and Abdolhossein PARnOW ${ }^{2}$ \\ ${ }^{1}$ Department of Exercise Physiology, General Directorate of Education in Basrah, Basrah, Iraq; ${ }^{2}$ Department of Exercise \\ Physiology, Faculty of Sport Sciences, Razi University, Kermanshah, Iran. \\ E-mail: muthnaalmfrjy@gmail.com
}

Objective. The present study sought to verify the effects of an exercise training on the memory along with the morphological assessment of the adrenal gland tissue in the rats with experimental autoimmune encephalomyelitis (EAE).

Methods. Female Lewis rats were randomly divided into three groups: EAE group, EAE group with exercise (EAE+Ex), and control group (CO). Each group contained 10 rats. To evaluate the memory, all rats were subjected to the Morris water maze learning test for four consecutive days and one day for a prop test. EAE was induced by guinea pig spinal cord homogenate emulsified in incomplete Freund's adjuvant and heat-mycobacterium. The exercise training on a motorized treadmill was initiated 3 weeks before EAE induction and disconnected 2 weeks post-induction.

Results. We found that exercise training for five weeks produced an improved swimming velocity related to memory improvement in EAE+Ex group in comparison with EAE group, but not an incurable adrenal gland tissue after EAE induction.

Conclusions. The experimental design selected for this study appears to be an effective treatment for memory in rats with experimental autoimmune encephalomyelitis.

Key words: exercise training, EAE model, memory, adrenal gland

The multiple sclerosis is an inflammatory demyelinating disease associated with neurological disability and cognitive impairment. Although the precise pathological mechanism of this disease is not well understood, the multiple sclerosis is known to be an immune-mediated disease, in which autoimmune $\mathrm{T}$ cells have been proposed to play a key role in patients suffering from this illness (Wootla et al. 2012). In the multiple sclerosis patients, the cognitive dysfunction is still a frequently reported disorder (Kurkowska-Jastrzebska et al. 2013). Approximately half of all patients with multiple sclerosis suffers from the learning and memory deficits (Mandolesi et al. 2010). The memory deficit is experienced in the earlyphase of the multiple sclerosis (Ziehn et al. 2010), when the hippocampus damage starts with a volume decrease in the CA1 region and then continues in the $\mathrm{CA} 2$ and $\mathrm{CA} 3$ regions and the dentate gyrus (Aharoni et al. 2019).

On other hand, the adrenal gland plays an important role in the multiple sclerosis due to its responses to stress that usually occurs during this disease (Reder et al. 1994). EAE animals are used as an experimental model of the multiple sclerosis since the etiology of EAE is basically the same as in the multiple sclerosis in human, represented by axonal deterioration and demyelization, cell death, and degradation of myelin basic protein (Bernardes et al. 2016).

In EAE, few studies have suggested that a decrease in the adrenal gland function led to susceptibility to increase and seriousness of the disease and that EAE 
have been assisted in the immune-endocrine response patterns to stress concept establishing (Heesen et al. 2007). The adrenal gland has been recognized to be a main component of the neuroendocrine responses to stress and it plays a critical role in the maintenance of the homeostasis (Smith and Vale 2006). It has been well determined that hormones of the adrenal gland are released during and after the stressful stimulation, mainly of that type used in evoking the learning. The degree, to which are these hormonal systems released depends on the intensity and the type of the compression employed (Bermudez-Rattoni 2007). The mode of the adrenal gland hormone production by physical training has been studied under normal conditions (Kyrou and Tsigos 2007), but not in the EAE model. Therefore, this study may be assigned as the first one that determines the effect of the exercise training on the adrenal gland in EAE model. In contrast, extensive evidence indicates that the adrenal gland is critically involved in the memory (De Kloet et al. 1999; McGaugh 2004), when releases the stress hormones.

Stress hormones affect the memory consolidation through the interactions with arousal-induced stimulation of noradrenergic mechanisms within the amygdala (Roozendaal et al. 2007). Conversely, the amygdala regulates the memory consolidation via its efferent projections to other brain regions (Roozendaal et al. 2007). The adrenal gland together with stress has also been recognized to influence the hippocampus at different levels (Kim et al. 2015). Therefore, both the adrenal gland defect and hippocampus dysfunction can elicit a memory impairment in the multiple sclerosis ailment.

Exercise training can be considered as a stress stimulus, which can upregulate the adrenal gland activity (Stults-Kolehmainen and Sinha 2014). It is characterized as a repetition of the exercise bouts over time, which results in an enhanced work capability (Glassman 2010) and has a close relationship to the cognitive function. The exercise training facilitates the angiogenesis, synaptogenesis, and neurogenesis via various cellular and molecular mechanism, which leads to memory and brain plasticity improvements (Mandolesi et al. 2018). The exercise training has been shown to specifically display a rise in neuroplasticity and an alteration in the gene expression related to the neural activation and regeneration (Tong et al. 2001). Laurin and co-workers (2001) have observed that the regular exercise training may alleviate the cognitive defections induced by dementia in elderly persons leading to an enhanced functional recovery in the
CNS post injuries. Rossi and co-workers (2009) have shown that the exercise training has a direct neuroprotective effect on the dendritic spine loss in striatal neurons of the EAE disease. The purpose of the present study was to find out how far the exercise training may improve the memory in the EAE rat model of the multiple sclerosis and affect the adrenal gland morphology.

\section{Materials and methods}

Animals. Thirty female Lewis rats 6-7 weeks old and weighing 100-125 g were used. They were received from the Animal Center Laboratory of Basra University of Medical Sciences, Iraq. The rats were housed in an environment with controlled temperature $\left(24 \pm 2{ }^{\circ} \mathrm{C}\right)$ and humidity $(60 \%)$, light with $12 \mathrm{~h} \mathrm{light/dark}$ cycles and had access to food and water ad libitum. The experiments were performed in accordance with the Guide for the Care and Use of Laboratory Animals (Institute for Laboratory Animal Research, National Research Council) and the Animal Ethics Committee of Basra University of Medical Sciences followed by the international rules for the animal research. When EAE was induced the rats were divided into three groups (10 rats/group): $\mathrm{EAE}+\mathrm{Exercise}(\mathrm{EAE}+\mathrm{Ex}), \mathrm{EAE}$, and control groups.

Estrous cycle assessment. As previously described (Rahn et al. 2014), the estrous cycle was monitored daily in female rats. The rats with the same day starting estrous cycle were selected. Vaginal lavage was performed between 7 and 8 a.m. using a gentle manual restraint. Vaginal excretion was taken with a plastic pipette filled with $10 \mu \mathrm{l}$ of normal saline $(0.9 \%)$ by entering the tip inside the animal vagina. The vaginal fluid was placed on the glass slides. One drop of the vaginal fluid was collected with a clean tip from every rat.

EAE induction and monitoring. EAE was induced as previously described by Donia et al. (2010). Briefly, the rats were injected with an inoculum containing $50 \mu \mathrm{g}$ guinea pig spinal cord homogenate emulsified in incomplete Freund's adjuvant (Sigma Aldrich, St. Louis, Missouri, United States) and $2 \mathrm{mg}$ of heatmycobacterium (Difco H37Ra). EAE was induced by injecting $50 \mu \mathrm{l}$ of the emulsion subcutaneously at the tail base. The signs of disability were monitored daily using EAE disease scores and body weight changes. The severity of EAE was scored as follows: 0 - no clinical signs; 1 - tail paralysis; 2 - hind limb paresis; 3 - both hind limbs paralysis: 4 - forelimbs paralysis; 5 - moribund or deceased (Kurkowska-Jastrzebska et al. 2013). 
Morris water maze tests (MWM). MWM is an effective test for the spatial learning and memory skills (Sun et al. 2015). The water maze tank was represented by a black circular pool $(136 \mathrm{~cm}$ diameter $\times$ $60 \mathrm{~cm}$ height) filled with water $\left(22 \pm 1^{\circ} \mathrm{C}\right)$ with a $25 \mathrm{~cm}$ of depth. The MWM was geographically divided into four quadrants (north, east, south, and west), which were located around the circumference of the tank. A circular platform was situated in the north-west quadrant submerged approximately $1.5 \mathrm{~cm}$ below the surface of the water. Each trial was recorded by a video camera linked to a monitoring scheme (EthoVision XT, Tehran, Iran), which was mounted above the pool. A tracking system was utilized to evaluate the time spent on the platform and the swimming speed until the rat reached the platform.

The rats were allowed to habituate to the apparatus for two days. The experiment consisted of a consecutive learning for four days and one day as prop day by a hidden platform detecting day. Beginning points varied in a quasi-random fashion so that in each trial the rats began from the location once and never started from the same place on the other day. Each rat was positioned facing the wall of the water pool at one of the four-set and allowed to swim to find invisible platform unobserved probe in the pool. During the each experiment, each rat was given up to $60 \mathrm{~s}$ to find the hidden platform.

Rats were generally guided to the platform by the investigator, otherwise if the rat reached the hidden platform, it was allowed to stay in the place for 30 s. If the rat was unable to find the platform within $60 \mathrm{~s}$, it was placed on it for the 30s during the first of the four days. On the fifth day, a prop test was performed, in which the platform was outside of the rat's reach. We repeated the prop test with a single trial without the platform after the last session of the exercise protocol.

Exercise training protocol. Treadmill exercise has been suggested to be more efficient in the neuroprotection by changing brain metabolism than the voluntary exercise (Hayes et al. 2008; Kinni et al. 2011). Therefore, the treadmill was chosen for the exercise training in this study. After habituation period, the exercise training protocol was initiated 3 weeks before EAE induction and continued for 2 weeks after EAE induction. The EAE+Ex group of rats run on the treadmill for $30 \mathrm{~min} /$ day, 5 days per week for a total of 5 weeks (Caliskan et al. 2019). The intensity started at a speed of $17 \mathrm{~m} / \mathrm{min}$ and was incrementally decreased $1 \mathrm{~m}$ per min for each subsequent week. A low level of electric shock $(0.3$ $\mathrm{mA}$ ) served to motivate the rats to run continuously.
Rats in the non-exercise groups were only placed on the treadmill track off.

Histological methods. After the prop test, the rats were anesthetized with ketamine/xylazine (3/1) and sacrificed. The adrenal gland was removed from the right side of the body and processed for the paraffin embedding by a standard histological procedure. The paraffin-embedded tissues were cut into $3 \mu \mathrm{m}$ thick sections by an ordinary microtome and mounted into silane-coated slides. The histological sections were stained with hematoxylin and eosin (H\&E) according to a general protocol.

Statisticalanalysis. International business machine SPSS 22.0 was used for all statistical analyses. The data are expressed as the mean \pm standard error of the mean. Data were analyzed using a one-way analysis of variance (ANOVA) with repeated measures followed by a protected LSD post hoc test. Significance was established at $\mathrm{p}<0.05$.

\section{Results}

Memory evaluation using the WMW. To assess spatial learning and memory, the swimming velocity was evaluated before and after the EAE induction, although the rats stimulated by EAE showed memory defects compared to the CO group. In contrast, compression among groups showed higher significance in the EAE+Ex ( $>0.01)$ compared to EAE group, as presented in the Figure 1. Albite CO group was significantly higher compared to EAE+Ex group $(p>0.002)$ as well as EAE group $(p<0.001)$, as shown in Figures 2A, 2B. The Figure 3 presents the statistical comparison of the control group of rats to other ones.

Adrenal gland. The light microscopic analysis showed morphological changes in the adrenal gland tissue in rats. Photomicrographs of H\&E stained sections of the adrenal gland in untrained control mice show the cortex and medulla regions with typical ultrastructural features (Figure 4). The EAE group shows tissue damage in the adrenal gland represented by cortex, inflammatory cells infiltration (red arrow), hyperemia of the spaces between medulla cells (black arrows) and degeneration of some medulla cells (Figure 5). Moreover, EAE+Ex shows an appearance of vacuoles, particularly evident in the cytoplasm of the adrenal cells (Figure 6).

\section{Discussion}

Memory includes processes that are used to acquire, store, retain, and later retrieve information. It is an important cognitive process, which is frequently 


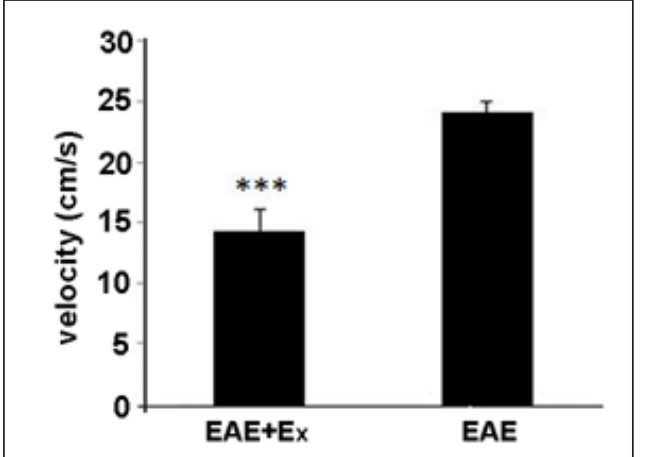

Figure 1. The graph shows significantly higher EAE+Ex in the speed velocity $(\mathrm{p}>0.01)$ compared to EAE group subjected to MWM test. Data are shown as group means $( \pm$ S.E.M $)$.

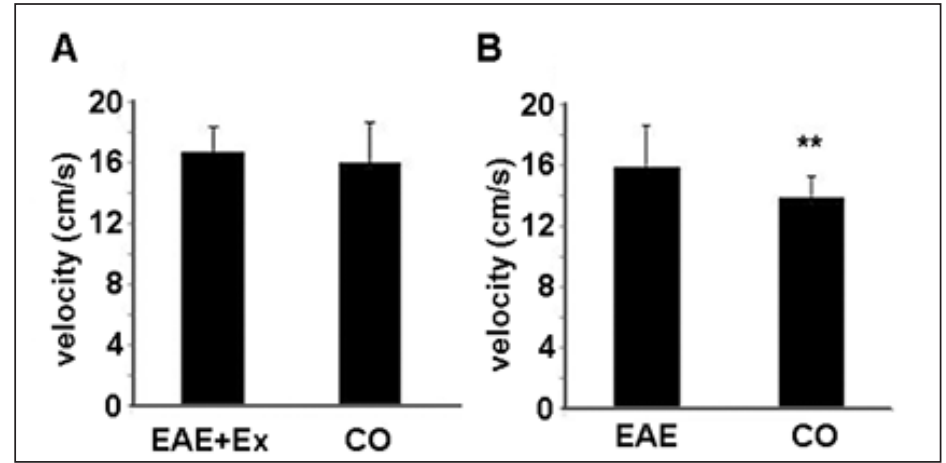

Figure 2. The graph shows significantly higher Co group in the speed velocity ( $>0.002$ ) compared to EAE+Ex (A) and EAE (B) groups. Data are shown as group means $( \pm$ S.E.M).

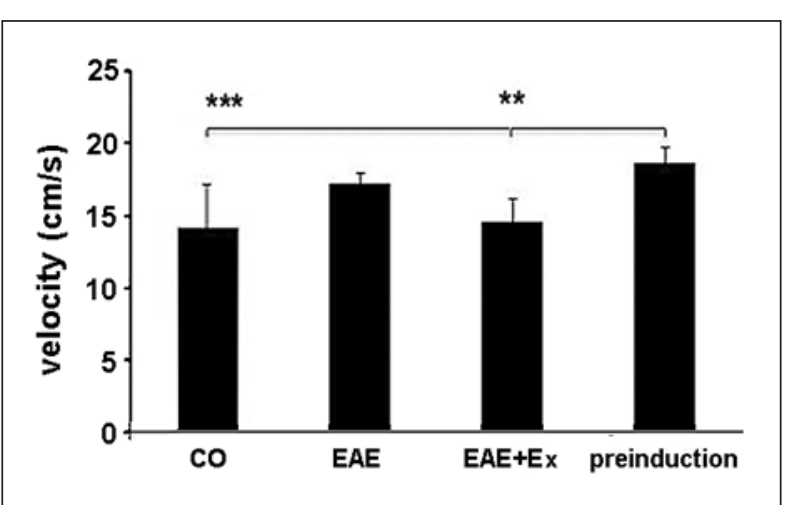

Figure 3. The graph shows significantly higher Co group in the speed velocity compared to other groups. Data are shown as group means $( \pm$ S.E.M $)$.

affected in the multiple sclerosis patients. Neuroimaging studies have established correlation between the multiple sclerosis and the cognitive dysfunction (Amato et al. 2019). Extensive evidence indicates that the adrenal gland is critically involved in the memory by releasing certain hormones (McIntyre and Roozendaal 2007). However, we did not find any studies related to memory with the adrenal gland morphology during the multiple sclerosis disease in humans or animals. Therefore, this study was aimed to examine the memory along with the adrenal gland tissue morphology under the exercise training for four weeks.

In this study, we found that the swimming velocity was increased in animals receiving EAE immunization subjected to regular treadmill exercise for 5 weeks. Our study was contacted with the previous study demonstrating that the memory can be improved by a regular exercise training program for 4 weeks in EAE (Kim and Sung 2017). In the past of the
EAE model, unexercised training studies have been showing memory loss noted in the EAE model on day 90 following induction instead of on day 30, which persisted till the completing the motor recovery (D'Intino et al. 2005). In contrast, a study of Sun and colleagues (2015) have determined that the learning and memory impairments were experienced in late $\mathrm{EAE}$ ( $55 \mathrm{dpi}$ ) rather than in the early stage ( $13 \mathrm{dpi}$ ) of the disease. Moreover, no memory deficits were observed in EAE animals compared to the control group on days 30 and $90 \mathrm{dpi}$, a findings suggesting that MS may start a neurodegenerative process in the hippocampus, which is not necessarily connected with the memory deficits (Kurkowska-Jastrzebska et al. 2013).

According to the conflicting data of unexercised training studies compared to exercise training data in the EAE model, we suggest that exercise is a stressor stimulus in humans as well as animals, which gives rise to several physiological changes that seek to bring an increase in the energy demand and also in the attaining of the homeostasis. It facilitates angiogenesis, synaptogenesis, and neurogenesis via different cellular and molecular processes, which lead to an improvement in the memory and brain plasticity (Mandolesi et al. 2018).

The present study demonstrates the adrenal gland induced damage in the tissue in both EAE and $\mathrm{EAE}+$ Exercise groups compared to the $\mathrm{CO}$ one. Our findings are aligned with the concept that the adrenal gland can be enlarged in the multiple sclerosis patients (Reder et al. 1994). An addition, the adrenal gland belongs to the hypothalamic-pituitary-adrenal (HPA) axis and it has been shown that the hormonal stress response is mainly coordinated by the HPA axis (Smith and Vale 2006). The multiple sclerosis and EAE induced dysregulation of the HPA axis (Heesen et al. 2007; Burfeind et al. 2016) resulted in a hypoactivity or 
upregulation of the glucocorticoid system including the secretion of corticosteroid hormones from the adrenal gland (Miler 2021). Glucocorticoids synthesis can promote HPA axis dysregulation with adverse impact on the accumulation of fat in the extremities, hypertension, skeletal muscle atrophy, and neurological defects (Gjerstad et al. 2018).

In the multiple sclerosis patients, medications with corticosteroids and dexamethasone are usually used and the EAE as model to modulate the HPA axis throughout the disease (Gold et al. 2012; Dos Santos et al. 2019). On other hand, Bartalucci and collaborators (2012) have inducted in non-EAE model studies that the distinct exercise training schedule of different intensity can cause changes in the mouse adrenal gland. Moreover, there are strong directories suggesting that the increase in the adrenal gland size after a stressful stimulation leads to an increase in the functional activity of the adrenal gland. This has been demonstrated by several studies, which have shown that following the exercise the functional activity of the adrenal cortex increasing leading to increased glucocorticoid levels, which is constantly accompanied by an increase in the adrenal gland whole size (Buuck et al. 1976). Although in the present study, using the EAE animal model, we could not find any improvement of 5 weeks of regular exercise training on the adrenal gland tissue, we found an unstructured damaging in the adrenal gland tissue. This is the first study where the adrenal gland tissue morphology was investigated in the EAE animal model that underwent exercise training. The comparison of the adrenal gland tissue in the EAE model with the non-EAE model does not give a realistic impression. Therefore, future investigations are necessary to evaluate the relationship between the adrenal gland of the EAE model and the exercise training related to the glucocorticoid system. In contrast, other exercise training protocols, such as chronic exercise for 9 weeks or 8 weeks, produced adrenal hypertrophy in the rat non-EAE model (Ring et al. 1970; Buuck and Tharp 1971).

The present data indicate that the exercise training-based protocol on duration in EAE animal model is incapable to reactivate the adrenal gland during the EAE disease due to the exercise training implicates to increase the width of zone fasciculate, which is responsible for decrease of the corticosteroids level. In the EAE model and during disease, the zone fasciculate hypertrophies accompanied to upregulation of the corticosteroids secretion and finally to damaging the adrenal gland tissue.

To our knowledge, this is the first study that examined the memory along with the adrenal gland

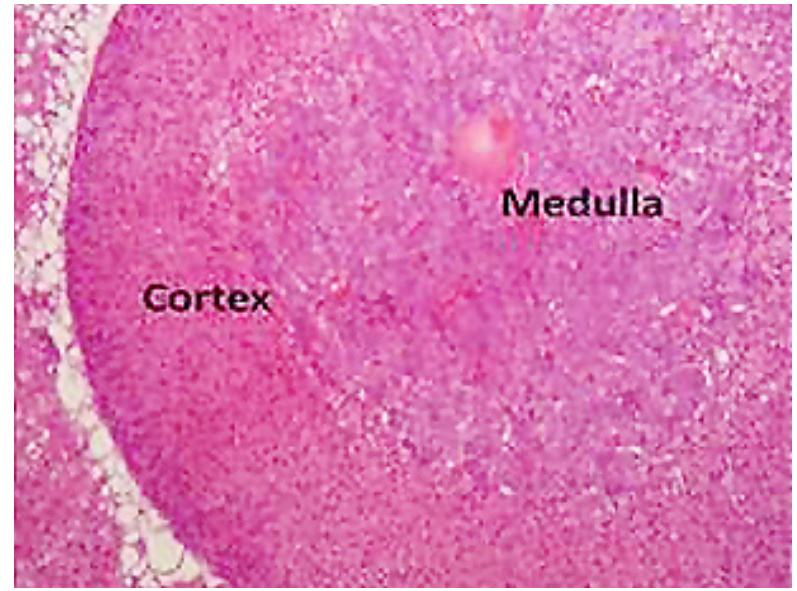

Figure 4. Histological section of the control group of rats showing normal structure of the adrenal gland cortex (Cortex) and medulla (Medulla). H\&E stain. $\times 10$.

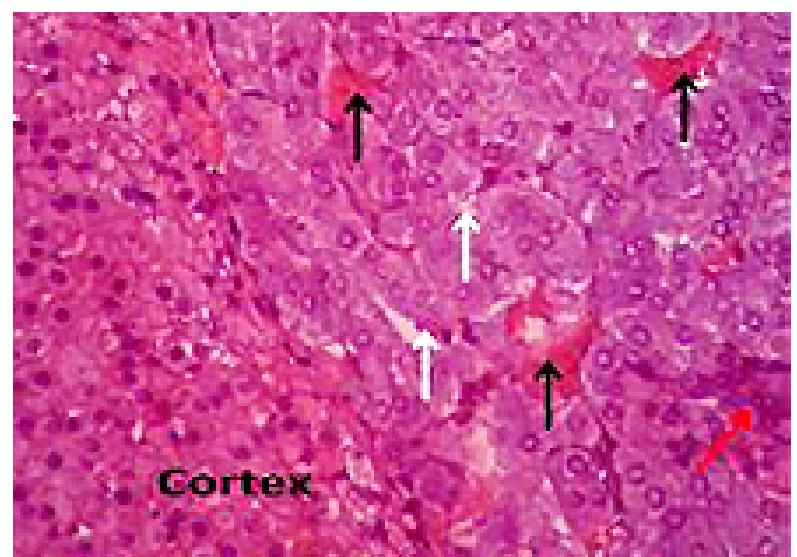

Figure 5. Histological section of the adrenal gland of Ex-EAE group showing the presence of vacuoles within the cytoplasm of the adrenal gland medulla cells (white arrow) and the occurrence of bleeding areas (black arrows). $\mathrm{H} \& \mathrm{E}$ stain. $\times 400$.

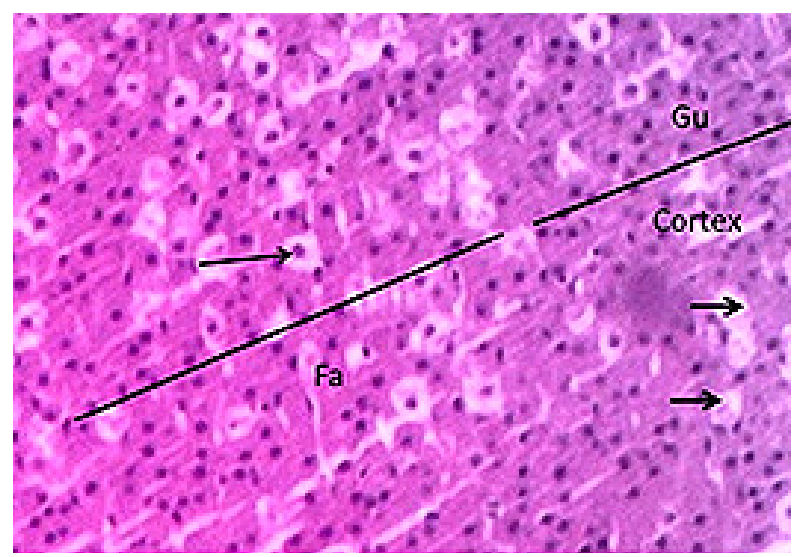

Figure 6. The adrenal gland from the EAE group showing the cortex, hyperemia of the spaces between the medulla cells (black arrows), and degeneration in some of the medulla fasciculata $(\mathrm{Fa})$ and glomerulosa $(\mathrm{Gu})$ zones cells. $\mathrm{H} \& \mathrm{E}$ stain. $\times 400$. 
tissue morphology in the EAE model affected by regular exercise training. The finding of the current study strongly indicates that the regular exercise training for 5 weeks may directly improve the memory impairment, but even though, the exercise protocol used herein did not incurable the adrenal gland tissue in the EAE model.

\section{Acknowledgment}

I thank to Dr. Fernando de Castro from Cajal-CSIC/Spanish Research Council for his support and Dr. Darpan I. Pateld from the School of Nursing, University of Texas, USA, for his help.

\section{References}

Aharoni R, Schottlender N, Bar-Lev DD, Eilam R, Sela M, Tsoory M, Arnon R. Cognitive impairment in an animal model of multiple sclerosis and its amelioration by glatiramer acetate. Sci Rep 9, 1-15, 2019.

Amato MP, Prestipino E, Bellinvia A, Niccolai C, Razzolini L, Pasto L, Fratangelo R, Tudisco L, Fonderico M, Mattiolo PL. Cognitive impairment in multiple sclerosis: An exploratory analysis of environmental and lifestyle risk factors. PloS One 14, e0222929, 2019.

Bartalucci A, Ferrucci M, Fulceri F, Lazzeri G, Lenzi P, Toti L, Serpiello FR, La Torre A, Gesi M. High-intensity exercise training produces morphological and biochemical changes in adrenal gland of mice. Histol Histopathol 27, 753-769, 2012.

Bermudez-Rattoni F. Neural Plasticity and Memory: From Genes to Brain Imaging, Boca Raton (FL), CRC Press/ Taylor and Francis, 2007.

Bernardes D, Brambilla R, Bracchi-Ricard V, Karmally S, Dellarole A, Carvalho-Tavares J, Bethea JR. Prior regular exercise improves clinical outcome and reduces demyelination and axonal injury in experimental autoimmune encephalomyelitis. J Neurochem 136, 63-73, 2016.

Burfeind KG, Yadav V, Marks DL. Hypothalamic dysfunction and multiple sclerosis: implications for fatigue and weight dysregulation. Curr Neurol Neurosci Rep 16, 1-11, 2016.

Buuck RJ, Tharp GD. Effect of chronic exercise on adrenocortical function and structure in the rat. J Appl Physiol 31, $880-883,1971$.

Buuck RJ, Tharp GD, Brumbaugh J. Effects of chronic exercise on the ultrastructure of the adrenocortical cells in the rat. Cell Tiss Res 168, 261-270, 1976.

Caliskan H, Akat F, Tatar Y, Zaloglu N, Dursun AD, Bastug M, Ficicilar H. Effects of exercise training on anxiety in diabetic rats. Behav Brain Res 376, 112084, 2019.

D’Intino G, Paradisi M, Fernandez M, Giuliani A, Aloe L, Giardino L, Calza L. Cognitive deficit associated with cholinergic and nerve growth factor down-regulation in experimental allergic encephalomyelitis in rats. Proc Nat Acad Sci 102, 3070-3075, 2005.

De Kloet ER, Oitzl MS, Joels M. Stress and cognition: are corticosteroids good or bad guys? Trends Neurosci 22, 422-426, 1999.

Donia M, Mangano K, Quattrocchi C, Fagone P, Signorelli S, Magro G, Sfacteria A, Bendtzen K, Nicoletti F. Specific and strain-independent effects of dexamethasone in the prevention and treatment of experimental autoimmune encephalomyelitis in rodents. Scand J Immunol 72, 396-407, 2010.

Dos Santos N, Novaes LS, Dragunas G, Rodrigues JR, Brandao W, Camarini R, Peron JPS, Munhoz CD. High dose of dexamethasone protects against EAE-induced motor deficits but impairs learning/memory in C57BL/6 mice. Sci Rep 9, 1-13, 2019.

Gjerstad JK, Lightman SL, Spiga F. Role of glucocorticoid negative feedback in the regulation of HPA axis pulsatility. Stress 21, 403-416, 2018.

Glassman G. Understanding CrossFit. East Valley Crossfit Newsletter 1, 1-115, 2010.

Gold SM, Sasidhar MV, Lagishetty V, Spence RD, Umeda E, Ziehn MO, Krieger T, Schulz K-H, Heesen C, Hewison M. Dynamic development of glucocorticoid resistance during autoimmune neuroinflammation. J Clin Endocrinol Met 97, E1402-E1410, 2012.

Hayes K, Sprague S, Guo M, Davis W, Friedman A, Kumar A, Jimenez DF, Ding Y. Forced, not voluntary, exercise effectively induces neuroprotection in stroke. Acta Neuropathol 115, 289-296, 2008.

Heesen C, Gold S, Huitinga I, Reul J. Stress and hypothalamic-pituitary-adrenal axis function in experimental autoimmune encephalomyelitis and multiple sclerosis - a review. Psychoneuroendocrinology 32, 604-618, 2007.

Kim EJ, Pellman B, Kim JJ. Stress effects on the hippocampus: a critical review. Learning Memory 22, 411-416, 2015.

Kim TW, Sung YH. Regular exercise promotes memory function and enhances hippocampal neuroplasticity in experimental autoimmune encephalomyelitis mice. Neuroscience 346, 173-181, 2017. 
Kinni H, Guo M, Ding JY, Konakondla S, Dornbos IIID, Tran R, Guthikonda M, Ding Y. Cerebral metabolism after forced or voluntary physical exercise. Brain Res 1388, 48-55, 2011.

Kurkowska-Jastrzebska I, Swiatkiewicz M, Zaremba M, Cudna A, Piechal A, Pyrzanowska J, Widy-Tyszkiewicz E, Czlonkowska A. Neurodegeneration and inflammation in hippocampus in experimental autoimmune encephalomyelitis induced in rats by one-time administration of encephalitogenic T cells. Neuroscience 248, 690-698, 2013.

Kyrou I, Tsigos C. Stress mechanisms and metabolic complications. Horm Metab Res 39, 430-438, 2007.

Laurin D, Verreault R, Lindsay J, MacPherson K, Rockwood K. Physical activity and risk of cognitive impairment and dementia in elderly persons. Arch Neurol 58, 498-504, 2001.

Mandolesi G, Grasselli G, Musumeci G, Centonze D. Cognitive deficits in experimental autoimmune encephalomyelitis: neuroinflammation and synaptic degeneration. Neurol Sci 31, 255-259, 2010.

Mandolesi L, Polverino A, Montuori S, Foti F, Ferraioli G, Sorrentino P, Sorrentino G. Effects of physical exercise on cognitive functioning and wellbeing: biological and psychological benefits. Front Psychol 9, 509, 2018.

McGaugh JL. The amygdala modulates the consolidation of memories of emotionally arousing experiences. Ann Rev Neurosci 27, 1-28, 2004.

McIntyre CK, Roozendaal B. Chapter 13. Adrenal Stress Hormones and Enhanced Memory for Emotionally Arousing Experiences. In: Bermudez-Rattoni F (Ed.). Neural Plasticity and Memory: From Genes to Brain Imaging. Boca Raton (FL), CRC Press/Taylor \& Francis, 2007.

Miler D. A closure looks on mechanism of corticosteroids. Ann Clin Lab Res 6, 64, 2021.

Rahn EJ, Iannitti T, Donahue RR, Taylor BK. Sex differences in a mouse model of multiple sclerosis: neuropathic pain behavior in females but not males and protection from neurological deficits during proestrus. Biology Sex Differ 5, 1-18, 2014.

Reder AT, Makowiec RL, Lowy MT. Adrenal size is increased in multiple sclerosis. Archives Neurol 51, 151-154, 1994.

Ring GC, Bosch M, Lo CS. Effects of exercise on growth, resting metabolism, and body composition of Fischer rats. Proc Soc Exp Biol Med 133, 1162-1165, 1970.

Roozendaal B, Barsegyan A, Lee S. Adrenal stress hormones, amygdala activation, and memory for emotionally arousing experiences. Progr Brain Res 167, 79-97, 2007.

Rossi S, Furlan R, De Chiara V, Musella A, Giudice TL, Mataluni G, Cavasinni F, Cantarella C, Bernardi G, Muzio L. Exercise attenuates the clinical, synaptic and dendritic abnormalities of experimental autoimmune encephalomyelitis. Neurobiol Dis 36, 51-59, 2009.

Smith SM, Vale WW. The role of the hypothalamic-pituitary-adrenal axis in neuroendocrine responses to stress. Dial Clin Neurosci 8, 383, 2006.

Stults-Kolehmainen MA, Sinha R. The effects of stress on physical activity and exercise. Sports Med 44, 81-121, 2014.

Sun JJ, Ren QG, Xu L, Zhang ZJ. LINGO-1 antibody ameliorates myelin impairment and spatial memory deficits in experimental autoimmune encephalomyelitis mice. Sci Rep 5, 1-12, 2015.

Tong L, Shen H, Perreau VM, Balazs R, Cotman CW. Effects of exercise on gene-expression profile in the rat hippocampus. Neurobiol Dis 8, 1046-1056, 2001.

Wootla B, Eriguchi M, Rodriguez M. Is multiple sclerosis an autoimmune disease? Autoimmune Dis 2012, 969657, 2012.

Ziehn MO, Avedisian AA, Tiwari-Woodruff S, Voskuhl RR. Hippocampal CA1 atrophy and synaptic loss during experimental autoimmune encephalomyelitis, EAE. Lab Invest 90, 774-786, 2010. 